FIgure

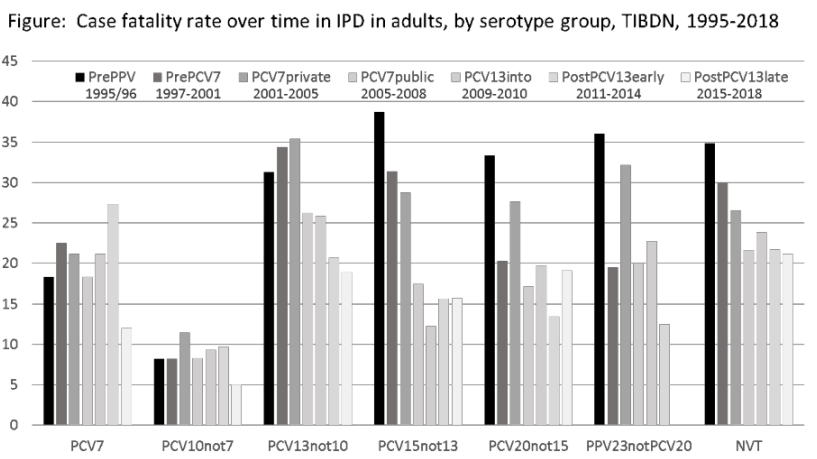

Conclusion: In our population, herd immunity from PCVs will result in a higher proportion of adult IPD occurring in immunocompromised cases, and a shift from bacteremic pneumonia to bacteremia without focus and meningitis.

Disclosures: Allison McGeer, MD, FRCPC, GlaxoSmithKline (Advisor or Review Panel member, Research Grant or Support)Merck (Advisor or Review Panel member, Research Grant or Support)Pfizer (Research Grant or Support)

296. IV-to-PO Antibiotic Step-down Therapy for Treatment of Uncomplicated Streptococcal Bloodstream Infections

Gerardo P. Ramos-Otero, PharmD ${ }^{1}$; Meghan Brett, $\mathrm{MD}^{1}$; Keenan L. Ryan, PharmD, $\mathrm{PhC}^{2}$; Preeyaporn Sarangarm, PharmD ${ }^{3}$; Carla Walraven, MS, PharmD, BCPS-AQ $\mathrm{ID}^{3} ;{ }^{1}$ University of New Mexico, San juan, Not Applicable, Puerto Rico; ${ }^{2}$ University of New Mexico Hospitals, Albuquerque, New Mexico ${ }^{3}$ University of New Mexico Hospital, Albuquerque, New Mexico

Session: P-9. Bacteremia

Background: Beta-lactams are the drug of choice for uncomplicated Streptococcal bloodstream infections (BSIs). However, due to the low bioavailability of oral beta-lactams, it's unclear whether de-escalation from IV to PO therapy is safe and effective. Our objective was to compare the efficacy and safety of step-down IV-to-PO antibiotic therapy to IV-only treatment of uncomplicated Streptococcal BSIs.

Methods: This was a retrospective study at a level-1, academic medical center in New Mexico of patients $\geq 18$ years of age treated for uncomplicated Streptococcal BSI between January 2017 and December 2019. The primary outcome was clinical failure in patients receiving IV-only therapy compared to IV-to-PO step-down therapy. Clinical failure was defined as having at least one of the following: persistent bacteremia, 30-day reinfection at any site or new-onset sepsis, 30-day BSI recurrence, or 30-day all-cause mortality. Secondary outcomes include 30-day all-cause readmission, 30-day antibiotic-related side effects, 30-day C. difficile-associated diarrhea and hospital length of stay (HLOS).

Results: A total of 98 patients were included: 51 in the IV-to-PO group and 47 in the IV-only therapy group. The median age for both groups was 61 years; $65 \%$ patients were male, and $72 \%$ were Caucasian. BSIs were predominantly associated with respiratory infections (24.5\%). Streptococcus pneumoniae (29.6\%) was the most common pathogen. Nine patients $(19.1 \%)$ in the IV-only group and none in the IV-to PO group experienced a clinical failure. 30-day reinfection at any site or new-onset sepsis $(88.9 \%)$ was the primary cause of clinical failures. Fourteen patients $(14.3 \%)$ were readmitted due to any cause within 30 days, 6 patients (11.8\%) from the IV-to-PO and 8 $(17 \%)$ from the IV-only group. Patients in the IV-to-PO group had a shorter duration of therapy than patients in the IV-only group ( 13 vs. 15 days, $\mathrm{p}=0.001$ ), and decreased HLOS ( 5 vs. 12 days, $\mathrm{p}<0.001$ ). Clinical failure was not statistically different when assessed for co-morbidities, source of infection, Pitt bacteremia score, documented BSI clearance, ICU admission or pathogen.

Conclusion: IV-to-PO step-down therapy appears to be a safe and effective alternative for treating uncomplicated Streptococcal BSIs in patients who are otherwise clinical stable.

Disclosures: Keenan L. Ryan, PharmD, PhC, Theravance (Advisor or Review Panel member)

\section{Lactobacillus: Friend or Foe}

Chiemeziem Nwanyanwu, MBBS ${ }^{1}$; Anuoluwapo Shobayo, $\mathrm{MD}^{2}$; Monica Ghitan, $\mathrm{MD}^{3}$; Edward Chapnick, $\mathrm{MD}^{3}$; Margaret Kuhn-Basti, $\mathrm{MD}^{3}$; Yu Shia Lin, $\mathrm{MD}^{3}$; Samuel Simon, PharmD ${ }^{4}$; Rosanna Li, PharmD ${ }^{3}$; ${ }^{1}$ Maimonides Medical Center, Brooklyn, NY, Brooklyn, New York ${ }^{2}$ Maimonides medical Center, Brooklyn, New York ${ }^{3}$ Maimonides Medical Center, Brooklyn, New York ${ }^{4}$ Maimondes Medical Center New York, NY

Session: P-9. Bacteremia

Background: Lactobacillus are low virulence commensal organisms which are commonly found in the human oral cavity, gastrointestinal and genitourinary tracts. Although Lactobacillus bacteremia (LB) is rare, evidence aggregating from case reports has implicated LB in several medical conditions. As such, there is reason to suggest that the presence of these organisms in blood cultures may not be due to spurious contamination, but rather, indicative of clinically meaningful events capable of inducing serious illnesses. The purpose of this study is to characterize the risk factors, clinical significance and outcomes of patients with LB.

Methods: We retrospectively reviewed the medical records of patients presenting to a large urban teaching hospital between January 1, 2017 and December 31, 2018, who were found to have LB. Identified individuals were grouped into two mutually exclusive case categories: true LB cases or non-true cases (i.e., contamination). Individuals with $\geq 1$ positive blood and were started on appropriate antibiotics were considered true cases. Those with positive cultures not started on appropriate antibiotics were considered contaminants.

Results: A total of 14 patients were identified during our study period, with majority considered true LB cases [71.4\%; $\mathrm{n}=10]$. These 14 individuals were mostly males $[64.2 \% ; \mathrm{n}=9]$ and reported no use of Lactobacilli probiotics $[78.6 \% ; \mathrm{n}=11]$ or antacids $[57.1 \% ; \mathrm{n}=8$ ]. On average, true LB cases were older (mean [SD]): 80.1 $[ \pm 10.9]$ vs. $54.0[ \pm 19.1]$ years $)$ and required longer hospitalization $(38.5[( \pm 27.6]$ vs. $8.0[( \pm 6.2]$ days $)$ compared to non-LB cases, respectively. Among the 10 true LB cases, the suspected source of infection included gastrointestinal system [50\%, $\mathrm{n}=5]$, infective endocarditis $[10 \% ; \mathrm{n}=1]$, genitourinary system $[10 \% ; \mathrm{n}=1]$; and could not be determined in 3 [30\%] cases. Concurrent infection with candida and gastrointestinal microbes were noted in four (40\%) of the true LB cases, respectively. Overall, five deaths were observed, with 4 [80\%] occurring in true LB cases and one in a non-LB case.

Conclusion: LB should not be dismissed as contaminants particularly in at-risk patients for LB, such as the elderly or immunocompromised individuals.

Disclosures: All Authors: No reported disclosures

298. Multicenter retrospective cohort study of the clinical significance of Staphylococcus lugdunensis isolated from a single blood culture set Naomi Hauser, MD, $\mathrm{MPH}^{1}$; Justin Kim, MD, $\mathrm{MPH}^{1}$; Paul Luethy, $\mathrm{PhD}^{2}$, Sarah Schmalzle, $\mathrm{MD}^{2}$; Jacqueline Bork, $\mathrm{MD}^{2} ;{ }^{1}$ University of Maryland Medical Center, Baltimore, Maryland; ${ }^{2}$ University of Maryland School of Medicine, Baltimore, MD

\section{Session: P-9. Bacteremia}

Background: Staphylococcus lugdunensis is a coagulase negative Staphylococcus (CoNS) species with the potential to cause aggressive infection. Guidance surrounding S. lugdunensis bacteremia (SLB) is lacking, especially in the case of a single positive set of blood cultures.

Methods: We performed a multicenter, retrospective observational cohort review of adult patients with SLB from at least one blood culture set within the University of Maryland Medical System from November 2015-November 2019. Objectives were to (1) describe baseline characteristics, (2) compare available criteria for evaluating clinical significance, and (3) evaluate the clinical outcomes among patients with SLB in 1 vs $\geq 2$ positive blood culture sets. Descriptive statistics with Chi-squared and MannWhitney $\mathrm{U}$ tests were carried out.

Results: There were 5,548 CoNS-positive blood culture sets, $49(0.88 \%)$ with S. lugdunensis comprising 36 adult patients ( 24 with 1 positive set and 12 with $\geq 2$ positive sets). Patients with $\geq 2$ positive sets were more likely to be on hemodialysis (HD) $(\mathrm{p}=0.029)$ and to have an HD catheter present $(\mathrm{p}=0.10)$ (Table 1). Thirty-five of the 36 patients fulfilled at least one of the following: systemic inflammatory response syndrome (SIRS), Souvenir criteria, or clinical criteria (infectious focus on imaging and/or second positive culture site) (Table 2). Twenty-eight (78\%) patients were treated with antimicrobial therapy and/or central line removal. SIRS criteria were met more often among patients with 1 positive set $(\mathrm{p}=0.05)$. Patients with $\geq 2$ positive sets were more often treated with antibiotics for longer than 2 weeks $(p=0.02)$. The mean time of positive cultures to discharge was 11 days and was longer for patients with only one set of positive blood cultures (13 vs. 6 days), although this difference was not statistically significant $(\mathrm{p}=0.29)$ (Table 3$)$.

\begin{tabular}{|c|c|c|c|c|}
\hline & \multicolumn{3}{|c|}{$\mathrm{N}(\%)$} & \multirow[b]{2}{*}{$\mathrm{p}$-value } \\
\hline & Total $(\mathrm{N}=36)$ & Single set $+(N=24)$ & $\geq 2$ set $+(N=12)$ & \\
\hline Female & $14(39)$ & $7(29)$ & $7(58)$ & 0.15 \\
\hline Age (median, IQR R $^{2}$ & $57.5(25.3)$ & $58(28)$ & $59(18)$ & 0.98 \\
\hline $\mathrm{BMI}^{\mathrm{b}}>24$ & \begin{tabular}{|l|}
$25(69)$ \\
\end{tabular} & $15(63)$ & $10(83)$ & 0.27 \\
\hline \multicolumn{5}{|l|}{ Comorbidities } \\
\hline Diabetes Mellitus & $15(42)$ & $9(38)$ & $6(50)$ & 0.50 \\
\hline Hypertension & $18(50)$ & $13(54)$ & $5(42)$ & 0.73 \\
\hline Hemodialysis & $7(19)$ & $2(8)$ & $5(42)$ & $0.029^{*}$ \\
\hline Malignancy & $7(19)$ & $4(17)$ & $3(25)$ & 0.66 \\
\hline Polymicrobial blood cultures & $19(53)$ & $14(58)$ & $5(42)$ & 0.48 \\
\hline Indwelling prosthetic material & $14(39)$ & $7(29)$ & $7(58)$ & 0.15 \\
\hline Joint & $4(11)$ & $3(13)$ & $1(8)$ & 1.0 \\
\hline $\mathrm{AICD} / \mathrm{PM}^{\mathrm{c}}$ & $4(11)$ & $3(13)$ & $1(8)$ & \\
\hline HD catheter ${ }^{d}$ & $4(11)$ & 1 (4) & $3(25)$ & $0.10^{*}$ \\
\hline Endovascular & $2(6)$ & 0 & $2(17)$ & 0.11 \\
\hline \multicolumn{5}{|c|}{$\begin{array}{l}\text { P values were calculated using the Fisher Exact Test for categorical variables and the Mann-Whitney } \mathrm{U} \text { test } \\
\text { for continuous variables. } \\
\text { * significant at } \mathrm{p} \leq 0.10 \\
\text { a Interquartile range } \\
\mathrm{b} \text { Body mass index } \\
\text { 'Automatic implantable cardioverter defibrillator/pacemaker } \\
\text { ¿ Hemodialysis }\end{array}$} \\
\hline
\end{tabular}




\begin{tabular}{|c|c|c|c|c|}
\hline & \multicolumn{3}{|c|}{$\mathrm{N}(\%)$} & \multirow[b]{2}{*}{$\mathrm{p}$-value } \\
\hline & Total $(\mathrm{N}=36)$ & Single set $+(N=24)$ & $\geq 2$ set $+(\mathrm{N}=12)$ & \\
\hline SIRS ${ }^{\mathrm{a}}$ criteria & $26(72)$ & $20(83)$ & $6(50)$ & 0.05 \\
\hline Souvenir criteria & $31(86)$ & $20(83)$ & $11(92)$ & 0.65 \\
\hline Clinical criteria & $12(33)$ & $7(29)$ & $5(42)$ & 0.48 \\
\hline Infection on imaging & $8(22)$ & $6(25)$ & $2(17)$ & 0.69 \\
\hline $2^{\text {nd }}$ culture site positive & $6(17)$ & $2(8)$ & $4(33)$ & 0.15 \\
\hline Intervention applied ${ }^{b}$ & $28(78)$ & $17(71)$ & $11(92)$ & 0.22 \\
\hline $\begin{array}{l}\text { P values were calculated using the Fis } \\
\text { a Systemic inflammatory response syr } \\
\text { b Antimicrobial therapy and/or centra }\end{array}$ & $\begin{array}{l}\text { ner Exact Test } \mathrm{f} \\
\text { drome } \\
\text { line removal }\end{array}$ & ir categorical variables & & \\
\hline
\end{tabular}

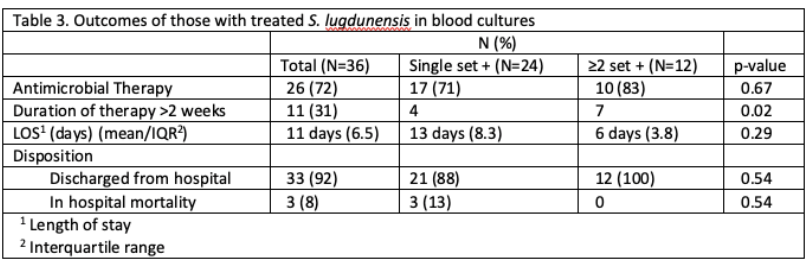

Conclusion: SLB was rare and occurred more frequently as a single set of positive blood cultures. Though limited by sample size, this study found similar patient characteristics, clinical significance and outcomes between patients with one set and those with $\geq 2$ sets of blood cultures positive for S. lugdunensis. Given the potential severity of SLB, it seems prudent to treat $S$. lugdunensis in a single blood culture, but larger studies are needed.

Disclosures: All Authors: No reported disclosures

299. Paediatric Collaborative Network on Infections in Canada (PICNIC) Study of the Current Landscape of Gram Negative Bacteremias

Alice X. Lu, n/a ${ }^{1}$; Kara Tsang, $\mathrm{PhD}(\mathrm{c})^{1}$; Michelle Barton, $\mathrm{MD}^{2}$; Craig Frankel, $\mathrm{MD}^{3}$; Jane McDonald, $\mathrm{MD}^{4}$; Jennifer Bowes, MSc ${ }^{5}$; John Gunawan, $\mathrm{MD}^{6}$; Sergio Fanella, MD, FRCPC, DTM\& $\mathrm{H}^{7}$; Mohammad Alghounaim, $\mathrm{MD}^{4}$; Jeannette Coumeau, $\mathrm{MD}^{8}$; Kirk Leifso, $\mathrm{MD}^{9}$; Robert Slinger, $\mathrm{MD}^{5}$; Joan Robinson, $\mathrm{MD}^{10}$; Sarah Khan, MD, MSc, FRCPC ${ }^{11}$; ${ }^{1}$ McMaster University, Hamilton, Ontario, Canada; ${ }^{2}$ Children's Hospital at London Health Centre, London, ON, Canada; ${ }^{3}$ Western University, London, Ontario, Canada; ${ }^{4}$ Montreal Children's Hospital, Montreal, QC, Canada; ${ }^{5}$ Children's Hospital of Eastern Ontario, Ottawa, ON, Canada; ${ }^{6}$ University of Alberta, Edmonton, Alberta, Canada; ${ }^{7}$ University of Manitoba, Winnipeg, MB, Canada; ${ }^{8}$ Dalhousie University, Halifax, Nova Scotia, Canada; ${ }^{9}$ Queen's University, Kingston, Ontario, Canada; ${ }^{10}$ Stollery Children's Hospital, Edmonton, AB, Canada; ${ }^{11}$ McMaster University, Hamilton, Ontario, Canada, Hamilton, Ontario, Canada

Pediatric Investigators Collaborative Network on Infections in Canada (PICNIC)

Session: P-9. Bacteremia

Background: Antimicrobial resistance is a public health threat, invasive infection from multi-drug resistant gram-negative (MDRGN) pathogens is associated with significant morbidity and mortality. The incidence of MDRGN bacteremia in Canada is rising, and pediatric data is limited.

Methods: This retrospective chart review of paediatric patients with gram negative bacteremia in a multicenter PICNIC database ( $\mathrm{n}=7$ centers) from 2013 to 2017 . MDRGN was defined as enterobacteriaceae that were resistant to third generation cephalosporins (including ESBL, CPE). Ethics approval was obtained at all sites, and data was entered into a secure REDCAP database, descriptive statistics are described herein.

Results: Of the 676 bacteremia patients in the database, $214(31.7 \%)$ were gram negative pathogens. E. coli was the most frequent pathogen $(59.8 \%$, of which 22 of 128 were MDR), followed by Klebsiella ( $31.8 \%$, of which 9 of 68 were MDR). Of the 31 MDRGNs, 19 were ESBL, 1 was a CPE, and 11 were nonspecific mechanisms of resistance. There were no multidrug resistant Pseudomonas, Stenotrophomonas, or Acinetobacter. The majority of patient were less than 3 months of age $(59.3 \%)$ and were male $(58.8 \%)$. The majority had an underlying comorbid condition; hematoncologic diagnosis accounting for $14.5 \%$. Length of stay varied from 1 to 742 days (mean 72 , standard deviation 88 ). 11\% required admission to ICU, $10 \%$ required removal of a intravascular catheter, $7 \%$ required a change in ventilation status, $2 \%$ requiring procedural source control, and there was an $8 \%$ mortality rate. Treatment duration greater than 14 days occurred in 123 patients (61\% of patients).

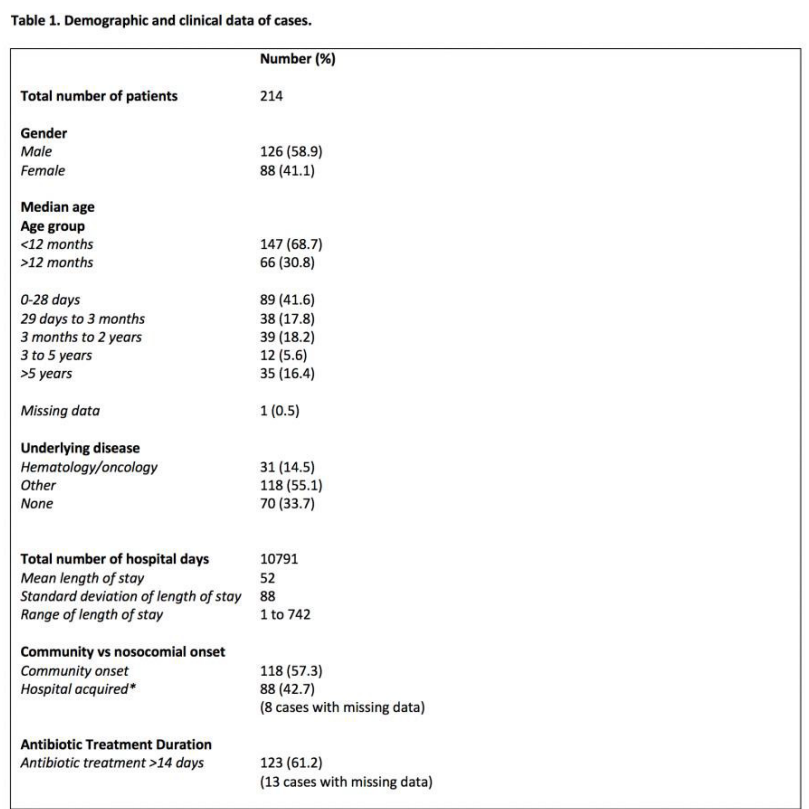

*Hospital acquired: first positive blood culture occurred after 72 hours into admission

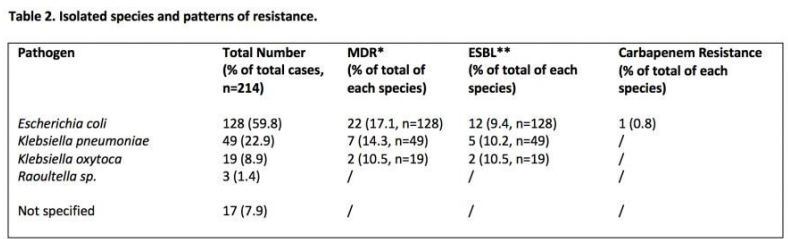

*Multidrug resistance (MDR) defined as having one of the following characteristics: ceftriaxone-resistant Enterobacteriaceae (indicative of ESBL producer); carbapenem-resistant Enterobacteriaceae (indicative of carbapenamase-producer);

**Extended-spectrum beta-lactamase (ESBL) defined as ceftriaxone-resistant Enterobacteriaceae.

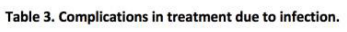

\begin{tabular}{|ll|}
\hline Characteristics & Number (\% of total cases, $\mathrm{n=214}$ ) (\% of complex, $\mathrm{n=165}$ ) \\
ICU odmission due to infection & $24(11.2)(14.5)$ \\
Removal of introvascular cotheter due to infection & $22(10.3)(13.3)$ \\
Change in ventilation requirements due to infection & $15(7.0)(9.1)$ \\
Infectious complication requiring surgical drainage & $4(1.9)(2.4)$ \\
Death & $18(8.4)(1.1)$ \\
Other complications due to infection & $31(14.5)(18.8)$ \\
\hline
\end{tabular}

Conclusion: This preliminary analysis of a multicenter review of pediatric gram negative bacteremias demonstrates a higher risk in neonates with comorbid conditions. A surprisingly prolonged treatment duration of greater than 14 days occurred in the majority of patients. Further analysis to assess factors associated with prolonged treatment durations, MDR infection, and complications is required. Gram negative bacteremia remains a significant cause of morbidity and mortality in pediatric patients.

Disclosures: All Authors: No reported disclosures

300. Pediatric Center Evaluation of the BioFire ${ }^{\oplus l o o d}$ Culture Identification 2 Panel Versus the Original BioFire ${ }^{\circ}$ FilmArray ${ }^{\circ}$ Blood Culture Identification Panel for the Detection of Microorganisms and Resistance Markers in Positive Blood Cultures

Kristina B. Pierce, $\mathrm{MS}^{1}$; Rebecca Barr, $\mathrm{MLS}^{2}$; Aubrie Hopper, $\mathrm{MLS}^{2}$;

Charlotte Bowerbank, n/a ${ }^{2}$; Anne Shaw, MLS²; J Pearson, MLS²; Matt Aldave, MLT²; 